\title{
On the Entropy Function and the Attractor Mechanism for Spherically Symmetric Extremal Black Holes
}

\author{
Rong-Gen Cai* \\ Institute of Theoretical Physics, Chinese Academy of Sciences, P.O. Box 2735, Beijing 100080, China \\ Li-Ming $\mathrm{CaO}^{\dagger}$ \\ Institute of Theoretical Physics, Chinese Academy of Sciences, P.O. Box 2735, Beijing 100080, China \\ Graduate School of the Chinese Academy of Sciences, Beijing 100039, China
}

\begin{abstract}
In this paper we elaborate on the relation between the entropy formula of Wald and the "entropy function" method proposed by A. Sen. For spherically symmetric extremal black holes, it is shown that the expression of extremal black hole entropy given by A. Sen can be derived from the general entropy definition of Wald, without help of the treatment of rescaling the $A d S_{2}$ part of near horizon geometry of extremal black holes. In our procedure, we only require that the surface gravity approaches to zero, and it is easy to understand the Legendre transformation of $f$, the integration of Lagrangian density on the horizon, with respect to the electric charges. Since the Noether charge form can be defined in an "off-shell" form, we define a corresponding entropy function, with which one can discuss the attractor mechanism for extremal black holes with scalar fields.
\end{abstract}

\footnotetext{
* e-mail address: cairg@itp.ac.cn

$\dagger$ e-mail address: caolm@itp.ac.cn
} 


\section{INTRODUCTION}

The attractor mechanism for extremal black holes has been studied extensively in the past few years in supergravity theory and superstring theory. It was initiated in the context supersymmetric BPS black holes [1, 2, 3, 4, 5, 6] and generalized to more general cases, such as supersymmetric black holes with higher order corrections [7, 8, 9, 10] and non-supersymmetric attractors [11, 12, 13, 14, 15].

Recently, A. Sen has proposed a so-called "entropy function" method for calculating the entropy of $n$-dimensional extremal black holes, where the extremal black holes are defined to be the spacetimes which have the near horizon geometry $A d S_{2} \times S^{n-2}$ and corresponding isometry [16, 17, 18, 19]. It states that the entropy of such kind of extremal black holes can be obtained by extremizing the "entropy function" with respect to some moduli on the horizon, where the entropy function is defined as $2 \pi$ times the Legendre transformation ( with respect to the electric charges ) of the integration of the Lagrangian over the spherical coordinates on the horizon in the near horizon field configurations. This method does not depend upon supersymmetry and has been applied or generalized to many solutions in supergravity theory, such as extremal black objects in higher dimensions, rotating extremal black holes, various non-supersymmetric extremal black objects and even near-extremal black holes $[20,21,22,23,24,25,26,27,28,29,30,31,32,33,34,35,36,37$, 38, 39, 40, 41].

In general, for spherically symmetric extremal black holes in a theory with Lagrangian $\mathcal{L}=$ $\mathcal{L}\left(g_{a b}, R_{a b c d}, \Phi_{s}, A_{a}^{I}\right)$, the near horizon geometry of these black holes has the form $A d S_{2} \times S^{n-2}$ [17, 18]. Due to $S O(1,2) \times S O(n-1)$ isometry of this geometry, the field configuration have the form as follows: The metric can be written down as

$$
d s^{2}=g_{a b} d x^{a} d x^{b}=v_{1}\left(-\rho^{2} d \tau^{2}+\frac{1}{\rho^{2}} d \rho^{2}\right)+v_{2} d \Omega_{n-2}^{2}
$$

where $v_{1}, v_{2}$ are constants which stand for the sizes of $A d S_{2}$ and $S^{n-2}$. Some other dynamical fields such as the scalar fields and $U(1)$ gauge fields are also taken to be constant: $\Phi_{s}=u_{s}$ and $F_{\rho \tau}^{I}=e_{I}$. The magnetic-type fields are also fixed with magnetic-charges $p_{i}$. Then, for this configuration, defining

$$
f\left(v_{1}, v_{2}, u_{s}, e_{I} ; p_{i}\right)=\int d x^{2} \wedge \cdots \wedge d x^{n-1} \sqrt{-g} \mathcal{L},
$$

where the integration is taken on the horizon, and $\left\{x^{2} \cdots x^{n-1}\right\}$ are angle coordinates of $S^{n-2}$, 
those constant moduli can be fixed via the equations of motion

$$
\frac{\partial f}{\partial v_{1}}=0, \quad \frac{\partial f}{\partial v_{2}}=0, \quad \frac{\partial f}{\partial u_{s}}=0, \quad \frac{\partial f}{\partial e_{I}}=q_{I},
$$

where $q_{I}$ are electrical-like charges for $U(1)$ gauge fields $A_{a}^{I}$. To relate the entropy of the black holes to these definitions, one defines $f_{\lambda}$ as (1.2) with the Riemann tensor part in $\mathcal{L}$ multiplied by a factor $\lambda$, and then one finds a relation between $f_{\lambda}$ and the Wald formula for spherically symmetric black holes [44]: $S_{B H}=-2 \pi \partial f_{\lambda} /\left.\partial \lambda\right|_{\lambda=1}$. Consider the structure of the Lagrangian, one can find

$$
\lambda \frac{\partial f_{\lambda}}{\partial \lambda}+v_{1} \frac{\partial f_{\lambda}}{\partial v_{1}}+e_{I} \frac{\partial f_{\lambda}}{\partial e_{I}}-f_{\lambda}=0 .
$$

When the equations of motion are satisfied, the entropy of black holes turns out to be $S_{B H}=$ $2 \pi\left(e_{I} q_{I}-f\right)$.

Therefore, one can introduce the "entropy function" for the extremal black holes

$$
\mathcal{E}\left(v_{1}, v_{2}, u_{s}, e_{I} ; p_{i}\right)=2 \pi\left(e_{I} q_{I}-f\left(v_{1}, v_{2}, u_{s}, e_{I} ; p_{i}\right)\right)
$$

which is obtained by carrying an integral of the Lagrangian density over $S^{n-2}$ and then taking the Legendre transformation with respect to the electric fields $e_{I}$. For fixed electric changes $q_{I}$ and magnetic charges $p_{i}$, these fields $u_{s}$ and $v_{1}$ and $v_{2}$ are determined by extremizing the entropy function with respect to the variables $u_{s}$ and $v_{1}$ and $v_{2}$. And then the entropy of the extremal black holes is given by the extremum of the entropy function by substituting the values of $v_{1}, v_{2}$ and $u_{s}$ back into the entropy function. In addition, let us notice that if the moduli fields $u_{s}$ are only dependent of the charges $q_{I}$ and $p_{i}$, the attractor mechanism is then manifested, and the entropy is a topological quantity.

This is a very simple and powerful method for calculating the entropy of such kind of extremal black holes. In particular, one can easily find the corrections to the entropy due to the higher derivative terms in the effective action. However, we notice that this method is established in a fixed coordinate system (1.1). If one uses another set of coordinates for the $A d S_{2}$ part, instead of the coordinates $\{\rho, \tau\}$, it seems that one can not define an entropy function as (1.5) because the function $f$ is not invariant under the coordinate transformation. In addition, the reason that to get the entropy of black holes, one should do the Legendre transformation with respect to the electric charges, but not include magnetic charges seems unclear in this procedure. Some authors have pointed out that the entropy function $\mathcal{E}$ resulting from this Legendre transformation of the function $f$ with respect to electric charges transforms as a function under the electric-magnetic dual, while the function $f$ does not [37]. But it is not easy to understand the Legendre transformation 
with respect to the angular-momentum $J$ in the rotating attractor cases [32]. There might be a more general formalism for the entropy function, and the Legendre transformation can be naturally understood in this frame. In this paper, we will elaborate these issues in the "entropy function" method and show that a general formalism of the "entropy function" method can be extracted from the black hole entropy definition due to Wald et al. [44, 45, 46]. In this procedure, we only require that the surface gravity of the black hole approaches to zero. Our entropy expression will reduce to the expression of A. Sen if we choose the same coordinates as in [17, 18].

The extremal black holes are different objects from the non-extremal ones due to different topological structures in Euclidean sector [49, 50, 51]. The extremal black hole has vanishing surface gravity and has no bifurcation surface, so the Noether charge method of Wald can not be directly used [44]. Thus, in this paper we regard the extremal black holes as the extremal limit of non-extremal black holes as in [17, 18, 42]. That is, we will first consider non-extremal black holes and then take the extremal limit. In this sense, the definitions of Wald are applicable.

The paper is organized as follows. In section II, we make a brief review on the entropy definition of Wald and give the required formulas. In section III, we give the near horizon analysis for the extremal black holes and derive the general form of the entropy. In section IV, we define the entropy function and discuss the attractor mechanism for the black holes with various moduli fields. The conclusion and discussion are given in section V.

\section{THE DEFINITION OF WALD}

In differential covariant theories of gravity, Wald showed that the entropy of a black hole is a kind of Noether charge [44, 45]. In this paper, we will use the Wald's method to define the entropy functions for spherically symmetric black holes. Assume the differential covariant Lagrangian of $n$-dimensional space-times $\left(M, g_{a b}\right)$ is

$$
\mathbf{L}=\mathcal{L}\left(g_{a b}, R_{a b c d}, \Phi_{s}, A_{a}^{I}\right) \boldsymbol{\epsilon},
$$

where we have put the Lagrangian in the form of differential form and $\epsilon$ is the volume element. $R_{a b c d}$ is Riemann tensor (since we are mainly concerning with extremal black holes, therefore we need not consider the covariant derivative of the Riemann tensor). $\left\{\Phi_{s}, s=0,1, \cdots\right\}$ are scalar fields, $\left\{A_{a}^{I}, I=1, \cdots\right\}$ are $U(1)$ gauge potentials, and the corresponding gauge fields are $F_{a b}^{I}=\partial_{a} A_{b}^{I}-\partial_{b} A_{a}^{I}$. We will not consider the Chern-Simons term as [18]. 
The variation of the Lagrange density $\mathbf{L}$ can be written as

$$
\delta \mathbf{L}=\mathbf{E}_{\psi} \delta \psi+d \mathbf{\Theta}
$$

where $\boldsymbol{\Theta}=\boldsymbol{\Theta}(\psi, \delta \psi)$ is an ( $n-1)$-form, which is called symplectic potential form, and it is a local linear function of field variation (we have denoted the dynamical fields as $\left.\psi=\left\{g_{a b}, \Phi_{s}, A_{a}^{I}\right\}\right) . \mathbf{E}_{\psi}$ corresponds to the equations of motion for the metric and other fields. Let $\xi$ be any smooth vector field on the space-time manifold, then one can define a Noether current form as

$$
\mathbf{J}[\xi]=\boldsymbol{\Theta}\left(\psi, \mathcal{L}_{\xi} \psi\right)-\xi \cdot \mathbf{L}
$$

where "." means the inner product of a vector field with a differential form, while $\mathcal{L}_{\xi}$ denotes the Lie derivative for the dynamical fields. A standard calculation gives

$$
d \mathbf{J}[\xi]=-\mathbf{E} \mathcal{L}_{\xi} \psi
$$

It implies that $\mathbf{J}[\xi]$ is closed when the equations of motion are satisfied. This indicates that there is a locally constructed $(n-2)$-form $\mathbf{Q}[\xi]$ such that, whenever $\psi$ satisfy the equations of motion, we have

$$
\mathbf{J}[\xi]=d \mathbf{Q}[\xi]
$$

In fact, the Noether charge form $\mathbf{Q}[\xi]$ can be defined in the so-called "off shell" form so that the Noether current $(n-1)$-form can be written as [46]

$$
\mathbf{J}[\xi]=d \mathbf{Q}[\xi]+\xi^{a} \mathbf{C}_{a},
$$

where $\mathbf{C}_{a}$ is locally constructed out of the dynamical fields in a covariant manner. When the equations of motion hold, $\mathbf{C}_{a}$ vanishes. For general stationary black holes, Wald has shown that the entropy of the black holes is a Noether charge [44], and may be expressed as

$$
S_{B H}=2 \pi \int_{\mathcal{H}} \mathbf{Q}[\xi]
$$

here $\xi$ be the Killing field which vanishes on the bifurcation surface of the black hole. It should be noted that the Killing vector field has been normalized here so that the surface gravity equals to "1". Furthermore, it was shown in [45] that the entropy can also be put into a form

$$
S_{B H}=-2 \pi \int_{\mathcal{H}} E_{R}^{a b c d} \epsilon_{a b} \epsilon_{c d},
$$

where $\boldsymbol{\epsilon}_{a b}$ is the binormal to the bifurcation surface $\mathcal{H}$, while $E_{R}^{a b c d}$ is the functional derivative of the Lagrangian with respect to the Riemann tensor with metric held fixed. This formula is purely 
geometric and does not include the surface gravity term. In this paper, since we will treat a limit procedure with surface gravity approaching to zero, we will not normalize the Killing vector such that the surface gravity equal to one. So we use the formula (2.8) to define the entropy of black holes as in 17, 18, 42]. For an asymptotically flat, static spherically symmetric black hole, one can simply choose $\xi=\partial_{t}=\frac{\partial}{\partial t}$.

For the Lagrangian as (2.1), we have

$$
\delta \mathbf{L}=\mathbf{E}^{a b} \delta g_{a b}+\mathbf{E}_{I}^{a} \delta A_{a}^{I}+\mathbf{E}^{s} \delta \Phi_{s}+d \mathbf{\Theta},
$$

where

$$
\begin{gathered}
\mathbf{E}_{I}^{a}=-2 \epsilon \nabla_{b}\left(\frac{\partial \mathcal{L}}{\partial F_{a b}^{I}}\right), \\
\mathbf{E}^{s}=\boldsymbol{\epsilon}\left(\frac{\partial \mathcal{L}}{\partial \Phi_{s}}-\nabla_{a} \frac{\partial \mathcal{L}}{\partial \nabla_{a} \Phi_{s}}\right), \\
\mathbf{E}^{a b}=\boldsymbol{\epsilon}\left(\frac{\partial \mathcal{L}}{\partial g_{a b}}+\frac{1}{2} g^{a b} \mathcal{L}+\frac{\partial \mathcal{L}}{\partial R_{c d e a}} R_{c d e}{ }^{b}+2 \nabla_{c} \nabla_{d} \frac{\partial \mathcal{L}}{\partial R_{\text {cabd }}}\right)
\end{gathered}
$$

are the equations of motion for the $U(1)$ gauge fields, the scalar fields and the metric $g_{a b}$, respectively. The symplectic potential form has the form

$$
\begin{aligned}
\boldsymbol{\Theta}_{a_{1} \cdots a_{n-1}} & =\left(\frac{\partial \mathcal{L}}{\partial \nabla_{a} \Phi_{s}} \delta \Phi_{s}+2 \frac{\partial \mathcal{L}}{\partial F_{a b}^{I}} \delta A_{b}^{I}\right. \\
& \left.+2 \frac{\partial \mathcal{L}}{\partial R_{a b c d}} \nabla_{d} \delta g_{b c}-2 \nabla_{d} \frac{\partial \mathcal{L}}{\partial R_{d b c a}} \delta g_{b c}\right) \boldsymbol{\epsilon}_{a a_{1} \cdots a_{n-1}} .
\end{aligned}
$$

Let $\xi$ be an arbitrary vector field on the space-time, The Lie derivative of $\xi$ on the fields are

$$
\mathcal{L}_{\xi} \Phi_{s}=\xi^{a} \nabla_{a} \Phi_{s}, \quad \mathcal{L}_{\xi} g_{a b}=\nabla_{a} \xi_{b}+\nabla_{b} \xi_{a}, \quad \mathcal{L}_{\xi} A_{a}^{I}=\nabla_{a}\left(\xi^{b} A_{b}^{I}\right)+\xi^{b} F_{b a}^{I}
$$

Substituting these Lie derivatives into the symplectic potential form, we find

$$
\begin{aligned}
\Theta_{a_{1} \cdots a_{n-1}} & =\left[\frac{\partial \mathcal{L}}{\partial \nabla_{a} \Phi_{s}} \xi^{b} \nabla_{b} \Phi_{s}+2 \frac{\partial \mathcal{L}}{\partial F_{a b}^{I}} \nabla_{b}\left(\xi^{c} A_{c}^{I}\right)+2 \frac{\partial \mathcal{L}}{\partial F_{a b}^{I}} \xi^{c} F_{c b}^{I}\right. \\
& \left.+2 \frac{\partial \mathcal{L}}{\partial R_{a b c d}} \nabla_{d}\left(\nabla_{b} \xi_{c}+\nabla_{c} \xi_{b}\right)-2 \nabla_{d} \frac{\partial \mathcal{L}}{\partial R_{d b c a}}\left(\nabla_{b} \xi_{c}+\nabla_{c} \xi_{b}\right)\right] \boldsymbol{\epsilon}_{a a_{1} \cdots a_{n-1}} \\
& =\left[\frac{\partial \mathcal{L}}{\partial \nabla_{a} \Phi_{s}} \xi^{b} \nabla_{b} \Phi_{s}+2 \nabla_{b}\left(\frac{\partial \mathcal{L}}{\partial F_{a b}^{I}} \xi^{c} A_{c}^{I}\right)-2 \nabla_{b} \frac{\partial \mathcal{L}}{\partial F_{a b}^{I}} \xi^{c} A_{c}^{I}+2 \frac{\partial \mathcal{L}}{\partial F_{a b}^{I}} \xi^{c} F_{c b}^{I}\right. \\
& \left.+2 \frac{\partial \mathcal{L}}{\partial R_{a b c d}} \nabla_{d}\left(\nabla_{b} \xi_{c}+\nabla_{c} \xi_{b}\right)-2 \nabla_{d} \frac{\partial \mathcal{L}}{\partial R_{d b c a}}\left(\nabla_{b} \xi_{c}+\nabla_{c} \xi_{b}\right)\right] \boldsymbol{\epsilon}_{a a_{1} \cdots a_{n-1}} .
\end{aligned}
$$


Then, we have

$$
\begin{aligned}
\boldsymbol{\Theta}_{a_{1} \cdots a_{n-1}} & =\left[2 \nabla_{b}\left(\frac{\partial \mathcal{L}}{\partial F_{a b}^{I}} \xi^{c} A_{c}^{I}\right)-\nabla_{b}\left(\frac{\partial \mathcal{L}}{\partial R_{a b c d}} \nabla_{[c} \xi_{d]}\right)\right] \boldsymbol{\epsilon}_{a a_{1} \cdots a_{n-1}}+\cdots \\
& +\left[\frac{\partial \mathcal{L}}{\partial \nabla_{a} \Phi_{s}} \xi^{b} \nabla_{b} \Phi_{s}+2 \frac{\partial \mathcal{L}}{\partial F_{a b}^{I}} \xi^{c} F_{c b}^{I}+\cdots \cdots\right] \boldsymbol{\epsilon}_{a a_{1} \cdots a_{n-1}} \\
& -2 \nabla_{b} \frac{\partial \mathcal{L}}{\partial F_{a b}^{I}} \xi^{c} A_{c}^{I} \boldsymbol{\epsilon}_{a a_{1} \cdots a_{n-1}}
\end{aligned}
$$

The first line in the above equation will give the Noether charge form, while the second line together with the terms in $\xi \cdot \mathbf{L}$ in Eq. (2.3) will give the constraint which corresponds to the equations of motion for the metric. For example, the first term in the second line combined with scalar fields terms in $\xi \cdot \mathbf{L}$ will give the energy-momentum tensor for scalar fields. Similarly the second term in the second line will enter the energy-momentum tensor for the $U(1)$ gauge fields in the equations of motion for the metric. The last line in the above equation will give the constraint which corresponds to the equations of motion for the $U(1)$ gauge fields. Thus, we find

$$
\mathbf{J}[\xi]=d \mathbf{Q}[\xi]+\xi^{a} \mathbf{C}_{a}
$$

where

$$
\mathbf{Q}=\mathbf{Q}^{F}+\mathbf{Q}^{g}+\cdots
$$

with

$$
\begin{gathered}
\mathbf{Q}_{a_{1} \cdots a_{n-2}}^{F}=\frac{\partial \mathcal{L}}{\partial F_{a b}^{I}} \xi^{c} A_{c}^{I} \boldsymbol{\epsilon}_{a b a_{1} \cdots a_{n-2}}, \\
\mathbf{Q}_{a_{1} \cdots a_{n-2}}^{g}=-\frac{\partial \mathcal{L}}{\partial R_{a b c d}} \nabla_{[c} \xi_{d]} \boldsymbol{\epsilon}_{a b a_{1} \cdots a_{n-2}} .
\end{gathered}
$$

The "..." terms are not important for our following discussion, so we brutally drop them at first. We will give a discussion at the end of the next section for these additional terms. Especially, the constraint for the $U(1)$ gauge fields is simply

$$
\mathbf{C}_{c a_{1} \cdots a_{n-1}}^{F}=-2 \nabla_{b} \frac{\partial \mathcal{L}}{\partial F_{a b}^{I}} A_{c}^{I} \boldsymbol{\epsilon}_{a a_{1} \cdots a_{n-1}}
$$

The term $\mathbf{Q}^{F}$ in the $\mathbf{Q}$ was not discussed explicitly in the earlier works of Wald et al. [44, 45, 46]. This is because that the killing vector vanishes on the bifurcation surface and the dynamical fields are assumed to be smooth on the bifurcation surface. However, in general, the $U(1)$ gauge fields are singular on the bifurcation surface, so one have to do a gauge transformation, $A \rightarrow A^{\prime}=A-\left.A\right|_{\mathcal{H}}$, 
such that the $\xi^{a} A_{a}^{\prime}$ are vanished on this surface, and then $\mathbf{Q}^{F}$. This gauge transformation will modify the data of gauge potential at infinity and an additional potential-charge term $\Phi \delta Q$ into the dynamics of the charged black holes from infinity, where $\Phi=\left.\xi^{c} A_{c}\right|_{\mathcal{H}}$ is the electrostatic potential on the horizon of the charged black hole and $Q$ is the electric charge [47]. Another treatment is: We only require the smoothness of the gauge potential projecting on the bifurcation surface, i.e., $\xi^{a} A_{a}$ instead of the gauge potential itself, so $\mathbf{Q}^{F}$ will generally not vanish on the bifurcation surface, and then $\Phi=\left.\xi^{c} A_{c}\right|_{\mathcal{H}}$ is introduced into the law of black hole without help of gauge transformation [48]. Similarly, in the next sections of this paper we only require that the projection of the gauge potential on the bifurcation surface is smooth. Since our final result will not depend on the gauge potential, the gauge transformation mentioned above will not effect our discussion. One can do such gauge transformation if necessary. In this paper, however, we will merely use the explicit form of the Noether charge $(n-2)$-form and we will not discuss the first law. Certainly, it is interesting to give a general discussion on the thermodynamics of these black holes. The relevant discussion can be found in a recent paper [43].

\section{ENTROPY OF EXTREMAL BLACK HOLES}

In this section, we will use the formulas above to give the general entropy function for static spherically symmetric extremal black holes. Assume that the metric for these black holes is of the form

$$
d s^{2}=-N(r) d t^{2}+\frac{1}{N(r)} d r^{2}+\gamma(r) d \Omega_{n-2}^{2},
$$

where $N, \gamma$ are functions of radial coordinate $r$, and $d \Omega_{n-2}^{2}$ is the line element for the $(n-2)$ dimensional sphere. The horizon $r=r_{H}$ corresponds to $N\left(r_{H}\right)=0$. If the equations of motion are satisfied, the constraint $\mathbf{C}_{a}=0$, and we have

$$
\mathbf{J}[\xi]=d \mathbf{Q}[\xi]
$$

Consider a near horizon region ranged from $r_{H}$ to $r_{H}+\Delta r$, we have

$$
\begin{aligned}
& \int_{r_{H}+\Delta r} \mathbf{Q}[\xi]-\int_{r_{H}} \mathbf{Q}[\xi]=\int_{\mathcal{H} \times \Delta r} \mathbf{J}[\xi] \\
& =\int_{\mathcal{H} \times \Delta r} \mathbf{\Theta}-\xi \cdot \mathbf{L} .
\end{aligned}
$$

If $\xi$ is a Killing vector, then $\Theta=0$, and

$$
\int_{r_{H}+\Delta r} \mathbf{Q}[\xi]-\int_{r_{H}} \mathbf{Q}[\xi]=-\int_{\mathcal{H} \times \Delta r} \xi \cdot \mathbf{L} .
$$


Thus we arrive at

$$
\begin{aligned}
& \int_{r_{H}+\Delta r} \mathbf{Q}^{g}[\xi]-\int_{r_{H}} \mathbf{Q}^{g}[\xi] \\
= & -\int_{r_{H}+\Delta r} \mathbf{Q}^{F}[\xi]+\int_{r_{H}} \mathbf{Q}^{F}[\xi]-\int_{\mathcal{H} \times \Delta r} \xi \cdot \mathbf{L} .
\end{aligned}
$$

Taking $\xi=\partial_{t}$, (since we consider the asymptotically flat space-time, $N(r)$ has the property $\lim _{r \rightarrow \infty} N(r)=1$, such that $\partial_{t}$ has a unit norm at infinity.), we have $\nabla_{[a} \xi_{b]}=\frac{1}{2} N^{\prime} \boldsymbol{\epsilon}_{a b}$, and

$$
\begin{aligned}
& \int_{r_{H}+\Delta r} \mathbf{Q}^{g}\left[\partial_{t}\right]-\int_{r_{H}} \mathbf{Q}^{g}\left[\partial_{t}\right] \\
= & \frac{1}{2}\left[N^{\prime}\left(r_{H}+\Delta r\right) B\left(r_{H}+\Delta r\right)-N^{\prime}\left(r_{H}\right) B\left(r_{H}\right)\right] \\
= & \frac{1}{2} \Delta r\left[N^{\prime \prime}\left(r_{H}\right) B\left(r_{H}\right)+N^{\prime}\left(r_{H}\right) B^{\prime}\left(r_{H}\right)\right]+\mathcal{O}\left(\Delta r^{2}\right),
\end{aligned}
$$

where we have defined a function $B(r)$

$$
B(r) \equiv-\int_{r} \frac{1}{(n-2) !} \frac{\partial \mathcal{L}}{\partial R_{a b c d}} \boldsymbol{\epsilon}_{c d} \boldsymbol{\epsilon}_{a b a_{1} \cdots a_{n-2}} d x^{a_{1}} \wedge \cdots \wedge d x^{a_{n-2}} .
$$

Note that the $\mathbf{Q}^{F}$ terms in the right hand side of Eq. (3.4) can be written as

$$
\begin{aligned}
& -\int_{r_{H}+\Delta r} \mathbf{Q}^{F}\left[\partial_{t}\right]+\int_{r_{H}} \mathbf{Q}^{F}\left[\partial_{t}\right] \\
= & A_{t}^{I}\left(r_{H}+\Delta r\right) q_{I}-A_{t}^{I}\left(r_{H}\right) q_{I} \\
= & q_{I} A_{t}^{\prime I}\left(r_{H}\right) \Delta r+\mathcal{O}\left(\triangle r^{2}\right) \\
= & q_{I} F_{r t}^{I}\left(r_{H}\right) \Delta r+\mathcal{O}\left(\triangle r^{2}\right)=q_{I} \tilde{e}_{I} \Delta r+\mathcal{O}\left(\triangle r^{2}\right),
\end{aligned}
$$

where $A_{t}^{I}=\left(\partial_{t}\right)^{a} A_{a}^{I}, \tilde{e}_{I} \equiv F_{r t}^{I}\left(r_{H}\right)$, and the $U(1)$ electrical-like charges are defined to be

$$
q_{I}=-\int_{r} \frac{1}{(n-2) !} \frac{\partial \mathcal{L}}{\partial F_{a b}^{I}} \epsilon_{a b a_{1} \cdots a_{n-2}} d x^{a_{1}} \wedge \cdots \wedge d x^{a_{n-2}}
$$

They do not change with the radii $r$. This is ensured by the Gaussian law. Note that there is an integration on the sphere part in (3.8), therefore the only $F_{r t}^{I}$ in $F_{a b}^{I}$ is relevant, so that we can simply write $F_{a b}^{I}\left(r_{H}\right)$ as $-\tilde{e}_{I} \boldsymbol{\epsilon}_{a b}$. Considering $-2 \tilde{e}_{I}^{2}=\tilde{e}_{I} \boldsymbol{\epsilon}_{a b} \tilde{e}_{I} \boldsymbol{\epsilon}^{a b}$ we have

$$
\frac{\partial \mathcal{L}}{\partial F_{a b}^{I}}=-\frac{\partial \mathcal{L}}{\partial \tilde{e}_{I}} \frac{\partial \tilde{e}_{I}}{\partial\left(\tilde{e}_{I} \boldsymbol{\epsilon}_{a b}\right)}=\frac{1}{2} \frac{\partial \mathcal{L}}{\partial \tilde{e}_{I}} \boldsymbol{\epsilon}^{a b}
$$

Substituting this result into the definition of the electric charges, we find

$$
q_{I}=-\frac{\partial}{\partial \tilde{e}_{I}} \int_{r_{H}} \frac{\mathcal{L}}{2(n-2) !} \epsilon^{a b} \boldsymbol{\epsilon}_{a b a_{1} \cdots a_{n-2}} d x^{a_{1}} \wedge \cdots \wedge d x^{a_{n-2}}=\frac{\partial \tilde{f}\left(r_{H}\right)}{\partial \tilde{e}_{I}} .
$$


Here $\tilde{f}\left(r_{H}\right)$ will be defined below in Eq. (3.12). The last term in the right hand side of Eq. (3.4) can be written as

$$
\int_{\mathcal{H} \times \Delta r} \partial_{t} \cdot \mathbf{L}=\int_{r_{H}}^{r_{H}+\Delta r} d r \int d x^{2} \wedge \cdots \wedge d x^{n-1} \sqrt{-g} \mathcal{L}=\int_{r_{H}}^{r_{H}+\Delta r} d r \tilde{f}(r),
$$

where

$$
\tilde{f}(r)=\int_{r} d x^{2} \wedge \cdots \wedge d x^{n-1} \sqrt{-g} \mathcal{L}
$$

Thus we arrive at

$$
\int_{\mathcal{H} \times \Delta r} \partial_{t} \cdot \mathbf{L}=\Delta r \tilde{f}\left(r_{H}\right)+\mathcal{O}\left(\triangle r^{2}\right)
$$

up to the leading order of $\triangle r$. Substituting Eqs. (3.5), (3.7) and (3.13) into Eq. (3.4), we get

$$
\begin{aligned}
& \frac{1}{2} \Delta r\left[N^{\prime \prime}\left(r_{H}\right) B\left(r_{H}\right)+N^{\prime}\left(r_{H}\right) B^{\prime}\left(r_{H}\right)\right]+\mathcal{O}\left(\Delta r^{2}\right) \\
= & \Delta r q_{I} \tilde{e}_{I}-\Delta r \tilde{f}\left(r_{H}\right) .
\end{aligned}
$$

Considering the limit $\Delta r \rightarrow 0$, we find

$$
\frac{1}{2}\left[N^{\prime \prime}\left(r_{H}\right) B\left(r_{H}\right)+N^{\prime}\left(r_{H}\right) B^{\prime}\left(r_{H}\right)\right]=q_{I} \tilde{e}_{I}-\tilde{f}\left(r_{H}\right) .
$$

So far, we have not specialized to extremal black holes; therefore, the above results hold for general non-extremal black holes. For the extremal black holes limit with $N^{\prime}\left(r_{H}\right) \rightarrow 0$, while $N^{\prime \prime}\left(r_{H}\right) \neq 0$, from (3.15) we have

$$
B\left(r_{H}\right)=\frac{2}{N^{\prime \prime}\left(r_{H}\right)}\left(q_{I} \tilde{e}_{I}-\tilde{f}\left(r_{H}\right)\right) .
$$

Since we view the extremal black holes as the extremal limit of non-extremal black holes, the entropy formula of Wald is applicable for the extremal black holes. Note that $B\left(r_{H}\right)$ is nothing but the integration in Eq. (2.8) without the $2 \pi$ factor. Thus, the entropy of the extremal black holes can be expressed as

$$
S_{B H}=2 \pi B\left(r_{H}\right)=\frac{4 \pi}{N^{\prime \prime}\left(r_{H}\right)}\left(q_{I} \tilde{e}_{I}-\tilde{f}\left(r_{H}\right)\right)
$$

This is one of main results in this paper. It is easy to see that this entropy form is very similar to the one in the "entropy function" method of A. Sen. But some remarks are in order:

(i). We have not stressed that the extremal black holes have the near horizon geometry $A d S_{2} \times S^{n-2}$ as in [17, 18] although the vanishing surface gravity and the metric assumption (3.1) may coincide with the definition through the near horizon geometry. However, let us notice that 
some extremal black holes have near horizon geometries of the form $A d S_{3}$ products some compact manifold $X$. In our procedure, the near horizon geometry is not necessary to be $A d S_{2} \times S^{n-2}$ and the only requirement is to have vanishing surface gravity. Therefore our procedure can be used to discuss that kind of extremal black holes whose near horizon geometry is of the form $A d S_{3} \times X$ by simply modifying the metric assumption in Eq.(3.1).

(ii). Our result is explicitly invariant under coordinate transformation, and this can be easily seen from the above process. We have not used the treatment method Eq.(1.4) employed by A. Sen.

(iii). The Legendre transformation with respect to the electric charges appears naturally in this procedure, while the Legendre transformation with respect to the magnetic charges does not appear.

(iv). If we choose a set of coordinates as the one in [17, 18], our expression for the entropy is exactly same as the one given by A. Sen. This can be seen as follows. In the extremal limit $N^{\prime}\left(r_{H}\right)=0$, we can rewrite the metric near the horizon as

$$
d s^{2}=-\frac{1}{2} N^{\prime \prime}\left(r_{H}\right)\left(r-r_{H}\right)^{2} d t^{2}+\frac{2}{N^{\prime \prime}\left(r_{H}\right)\left(r-r_{H}\right)^{2}} d r^{2}+\gamma\left(r_{H}\right) d \Omega_{n-2}^{2} .
$$

Redefine the coordinates as

$$
\rho=r-r_{H}, \quad \tau=\frac{1}{2} N^{\prime \prime}\left(r_{H}\right) t
$$

Then, the near horizon metric can be further rewritten as

$$
d s^{2}=\frac{2}{N^{\prime \prime}\left(r_{H}\right)}\left(-\rho^{2} d \tau^{2}+\frac{1}{\rho^{2}} d \rho^{2}\right)+\gamma\left(r_{H}\right) d \Omega_{n-2}^{2} .
$$

The components of gauge fields $F_{r t}^{I}$ and $\tilde{f}$ are dependent of coordinates, in this new set of coordinates they are

$$
\begin{gathered}
\tilde{e}_{I}=\frac{1}{2} N^{\prime \prime}\left(r_{H}\right) e_{I}, \\
\tilde{f}\left(r_{H}\right)=\frac{1}{2} N^{\prime \prime}\left(r_{H}\right) f .
\end{gathered}
$$

where

$$
e_{I}=F_{\rho \tau}^{I}\left(r_{H}\right), \quad f=\int_{r_{H}} d x^{2} \wedge \cdots \wedge d x^{n-1} \sqrt{-g^{\prime}} \mathcal{L} .
$$

Since the entropy is invariant under the coordinate transformation, we find in these coordinates like $\{\tau, \rho, \cdots\}$,

$$
S_{B H}=2 \pi\left(q_{I} e_{I}-f\right) .
$$


This is nothing but the entropy formula given by A. Sen for extremal black holes. Since the factor $2 / N^{\prime \prime}\left(r_{H}\right)$ in (3.17) disappears in this new set of coordinates, the entropy formula becomes more simple and good look. This is an advantage of this set of coordinates. But we would like to stress that the entropy expression with the factor " $2 / N^{\prime \prime}\left(r_{H}\right)$ " makes it invariant under coordinate transformation.

(v). Finally the function $\tilde{f}\left(r_{H}\right)$ is evaluated for the solution of the equations of motion, i.e. all the fields: $\left\{g_{a b}, \Phi_{s}, F_{a b}^{I}\right\}$ are on shell. For example, if the near horizon geometry has the form

$$
d s^{2}=v_{1}\left(-\rho^{2} d \tau^{2}+\frac{1}{\rho^{2}} d \rho^{2}\right)+v_{2} d \Omega_{n-2}^{2},
$$

and the equations of motion are satisfied, then we can express the entropy in the form (3.24). There $v_{1}$ and $v_{2}$ should equal to $2 / N^{\prime \prime}\left(r_{H}\right)$ and $\gamma\left(r_{H}\right) . N, \gamma$, and other fields, should satisfy the equations of motion.

One may worry about that the conserved charge form $\mathbf{Q}$ in Eq.(2.18) is not complete: For example, we will have an additional term $\boldsymbol{\epsilon}_{a b a_{1} \cdots a_{n-2}} \xi^{a} \nabla^{b} D(\phi)$ if the action has a dilaton coupling term $D(\phi) R$. In general, the conserved charge form can be written as $\mathbf{Q}=\mathbf{Q}^{F}+\mathbf{Q}^{g}+\xi^{a} \mathbf{W} a+\mathbf{Y}+d \mathbf{Z}$, where $\mathbf{W}_{a}, \mathbf{Y}$ and $\mathbf{Z}$ are smooth functions of fields and their derivatives, and $\mathbf{Y}=\mathbf{Y}\left(\psi, \mathcal{L}_{\xi} \psi\right)$ is linear for the field variation [45, 46]. Obviously, $\mathbf{Y}$ and $d \mathbf{Z}$ will not give contributions to the near horizon integration (3.2) if $\xi$ is a killing vector. It seems that $\xi^{a} \mathbf{W}_{a}$ will give an additional contribution to this integration. For the extremal case, this contribution will vanish due to the smoothness of $\mathbf{W}_{a}$ and the vanishing surface gravity. For example, the term corresponding to the dilaton coupling mentioned above will vanish in the near horizon integration. So the final form of the entropy (3.17) will not change. For the non-extremal case, this term essentially appear in the near horizon integration if we add the $\xi^{a} \mathbf{W}_{a}$ into $\mathbf{Q}$. However, if necessary, we can always change the Lagrangian $\mathbf{L}$ to be $\mathbf{L}+d \boldsymbol{\mu}$ and put the conserved charge form $\mathbf{Q}$ into the form of (2.18) without the "..." terms, where $\boldsymbol{\mu}$ is a $(n-1)$-form. This change of Lagrangian will not affect the equations of motion and the entropy of the black holes [45, 46]. Then, the formulas (3.4) and therefore (3.15) are still formally correct for the non-extremal case after considering that ambiguity of the Lagrangian and therefore $\tilde{f}\left(r_{H}\right)$. But this ambiguity has no contribution to Eq. (3.17) which describes the entropy of the black hole in the extremal case. 


\section{ENTROPY FUNCTION AND ATTRACTOR MECHANISM}

In this section we show further that one can define an entropy function with the help of the entropy definition of Wald. The Noether current can always be written as $\mathbf{J}[\xi]=d \mathbf{Q}[\xi]+\xi^{a} \mathbf{C}_{a}$ where $\mathbf{C}_{a}$ corresponds to constraint. The constraint for the $U(1)$ gauge fields is (2.21). If the equations of motion for the $U(1)$ gauge fields hold, this constraint vanishes. In this section, we will assume the equations of motion for the $U(1)$ gauge fields are always satisfied, but not for the metric and scalar fields. In other word, we will not consider the constraint for the gauge fields. Assuming that the metric of the extremal black holes has the form

$$
d s^{2}=-N(r) d t^{2}+\frac{1}{N(r)} d r^{2}+\gamma(r) d \Omega_{n-2}^{2},
$$

on the horizon $r=r_{H}$ of an extremal black hole, one has $N\left(r_{H}\right)=0, N^{\prime}\left(r_{H}\right)=0$, but $N^{\prime \prime}\left(r_{H}\right) \neq 0$. Thus the near horizon geometry will be fixed if $N^{\prime \prime}\left(r_{H}\right)$ and $\gamma\left(r_{H}\right)$ are specified. This means the "off-shell" of the near horizon geometry corresponds to the arbitrariness of the parameter $N^{\prime \prime}\left(r_{H}\right)$ and $\gamma\left(r_{H}\right)$. In the near horizon region ranged from $r_{H}$ to $r_{H}+\Delta r$, we have

$$
\begin{aligned}
& \int_{r_{H}+\Delta r} \mathbf{Q}[\xi]-\int_{r_{H}} \mathbf{Q}[\xi]+\int_{\mathcal{H} \times \Delta r} \xi^{a} \mathbf{C}_{a} \\
& =\int_{\mathcal{H} \times \Delta r} \mathbf{J}[\xi]=\int_{\mathcal{H} \times \Delta r} \mathbf{\Theta}-\xi \cdot \mathbf{L} .
\end{aligned}
$$

If $\xi$ is a Killing vector for the field configuration space for our discussion (the solution space is a subset of this space), then $\Theta=0$, and we have

$$
\int_{r_{H}+\Delta r} \mathbf{Q}[\xi]-\int_{r_{H}} \mathbf{Q}[\xi]+\int_{\mathcal{H} \times \Delta r} \xi^{a} \mathbf{C}_{a}=-\int_{\mathcal{H} \times \Delta r} \xi \cdot \mathbf{L} .
$$

With this, we obtain

$$
\begin{aligned}
& \int_{r_{H}+\Delta r} \mathbf{Q}^{g}[\xi]-\int_{r_{H}} \mathbf{Q}^{g}[\xi]+\int_{\mathcal{H} \times \Delta r} \xi^{a} \mathbf{C}_{a} \\
= & -\int_{r_{H}+\Delta r} \mathbf{Q}^{F}[\xi]+\int_{r_{H}} \mathbf{Q}^{F}[\xi]-\int_{\mathcal{H} \times \Delta r} \xi \cdot \mathbf{L} .
\end{aligned}
$$

Define our "entropy function" as

$$
\mathcal{E}=\lim _{\Delta r \rightarrow 0} \frac{4 \pi}{N^{\prime \prime}\left(r_{H}\right) \Delta r}\left\{\int_{r_{H}+\Delta r} \mathbf{Q}^{g}\left[\partial_{t}\right]-\int_{r_{H}} \mathbf{Q}^{g}\left[\partial_{t}\right]+\int_{\mathcal{H} \times \Delta r}\left(\partial_{t}\right)^{a} \mathbf{C}_{a}\right\} .
$$

If the equations of motion are satisfied, obviously, this $\mathcal{E}$ will reduce to the entropy of extremal black holes given in the previous section. Therefore this definition is meaningful. Further, from Eq. (4.3), we have

$$
\mathcal{E}=\lim _{\Delta r \rightarrow 0} \frac{4 \pi}{N^{\prime \prime}\left(r_{H}\right) \Delta r}\left\{-\int_{r_{H}+\Delta r} \mathbf{Q}^{F}\left[\partial_{t}\right]+\int_{r_{H}} \mathbf{Q}^{F}\left[\partial_{t}\right]-\int_{\mathcal{H} \times \Delta r} \partial_{t} \cdot \mathbf{L}\right\} .
$$


Recalling that the equations of motion for the $U(1)$ gauge fields have been assumed to hold always, and following the calculations in the previous section, we have

$$
\mathcal{E}=\frac{4 \pi}{N^{\prime \prime}\left(r_{H}\right)}\left(\tilde{e}_{I} q_{I}-\tilde{f}\left(r_{H}\right)\right)
$$

This expression looks the same as the one given in the previous section. However, a crucial difference from the one in the previous section is that here the fields need not be the solutions of the equations of motion. To give the entropy of the extremal black holes, we have to solve the equations of motion or extremize the entropy function with respect to the undetermined values of fields on the horizon. It is easy to find that entropy function has the form

$$
\mathcal{E}=\mathcal{E}\left(N^{\prime \prime}, \gamma, u_{s}, \tilde{e}_{I} ; p_{i}\right)=\frac{4 \pi}{N^{\prime \prime}}\left(\tilde{e}_{I} q_{I}-\tilde{f}_{H}\left(N^{\prime \prime}, \gamma, u_{s}, \tilde{e}_{I} ; p_{i}\right)\right)
$$

where, for simplicity, we have denoted the $N^{\prime \prime}\left(r_{H}\right)$ and $\gamma\left(r_{H}\right)$ by $N^{\prime \prime}$ and $\gamma$, respectively. The terms $u_{s}^{\prime}$ will not appear because those kinetic terms of scalar fields in the action always have a vanishing factor $N\left(r_{H}\right)=0$ on the horizon. Similarly, $\gamma^{\prime}\left(r_{H}\right), \gamma^{\prime \prime}\left(r_{H}\right)$ will not appear because that the components of the Riemann tensor which include these terms have to contract with the vanished factors $N\left(r_{H}\right)$ or $N^{\prime}\left(r_{H}\right)$. Certainly, this point can be directly understood from the near horizon geometry in Eq. (3.20). So, extremizing the entropy function becomes

$$
\frac{\partial \mathcal{E}}{\partial N^{\prime \prime}}=0, \quad \frac{\partial \mathcal{E}}{\partial \gamma}=0, \quad \frac{\partial \mathcal{E}}{\partial u_{s}}=0 .
$$

The electric charges are determined by

$$
\frac{\partial \mathcal{E}}{\partial \tilde{e}_{I}}=0 \quad \text { or } \quad q_{I}=\frac{\partial \tilde{f}\left(r_{H}\right)}{\partial \tilde{e}_{I}} .
$$

The entropy of the black hole can be obtained by solving these algebraic equations, and substituting the solutions for $N^{\prime \prime}, \gamma, u_{s}$ back into the entropy function. If the values of moduli fields on the horizon are determined by charges of black holes, then the attractor mechanism is manifest. Then the entropy has the form

$$
S_{B H}=S_{B H}\left(q_{I} ; p_{i}\right)=\left.\mathcal{E}\right|_{\text {extremum piont }},
$$

a topological quantity which is fully determined by charges [17, 18]. These definitions will become more simple if one chooses the coordinates $\{\tau, \rho, \cdots\}$ so that one can define

$$
v_{1}=\frac{2}{N^{\prime \prime}\left(r_{H}\right)}, \quad v_{2}=\gamma\left(r_{H}\right),
$$

then, the entropy function can be written as

$$
\mathcal{E}=\mathcal{E}\left(v_{1}, v_{2}, u_{s}, e_{I} ; p_{i}\right)=2 \pi\left(e_{I} q_{I}-f\left(v_{1}, v_{2}, u_{s}, e_{I} ; p_{i}\right)\right)
$$


where $e_{I}$ are gauge fields on the horizon in this set of coordinates, and $q_{I}=\frac{\partial f}{\partial e_{I}}$ are electric charges which are not changed with the coordinate transformation. So, in this set of coordinates, our entropy function form reduces to the entropy function defined by A. Sen [17, 18].

\section{CONCLUSION AND DISCUSSION}

In this paper, we have shown that the "entropy function" method proposed by A. Sen can be extracted from the general black hole entropy definition of Wald [44]. For a spherically symmetry extremal black hole as described by metric (3.1), we find that the entropy of the black hole can be put into a form

$$
S_{B H}=\frac{4 \pi}{N^{\prime \prime}\left(r_{H}\right)}\left(\tilde{e}_{I} q_{I}-\tilde{f}\left(r_{H}\right)\right)
$$

which is similar to the one given in Ref. [17, 18]. To get this entropy form, we have regarded the extremal black hole as the extremal limit of an non-extremal black hole, i.e., we have required (and only required) that the surface gravity approaches to zero. In a special set of coordinates, i.e., $\{\tau, \rho \cdots\}$, this entropy is exactly of the same form as the one given by A. Sen. We have obtained a corresponding entropy function (4.7). After extremizing this entropy function with respect to $N^{\prime \prime}, \gamma$ and other scalar fields, one gets the entropy of the extremal black holes. Similarly, in the coordinates $\{\tau, \rho \cdots\}$, our entropy function reduces to the form of A. Sen. Note that in our procedure, we have neither used the treatment of rescaling $A d S_{2}$ part of the near horizon geometry of extremal black holes, nor especially employed the form of the metric in the coordinates $\{\tau, \rho, \cdots\}$ as Eq.(1.1). In this procedure, it can be clearly seen why the electric charge terms $e_{I} q_{I}$ appear, but not the magnetic charges terms in the entropy function.

Recently it was shown that for some near-extremal black holes with BTZ black holes being a part of the near horizon geometry, that the "entropy function" method works as well [40]. A similar discussion for non-extremal D3, M2 and M5 branes has also been given in [41]. Therefore it is interesting to see whether the procedure developed in this paper works or not for near-extremal black holes. In this case, $N^{\prime}\left(r_{H}\right)$ is an infinitesimal one instead of vanishing. Eq. (3.15) then gives

$$
S_{B H}=2 \pi B\left(r_{H}\right)=S_{0}\left(1+\frac{N^{\prime}\left(r_{H}\right)}{N^{\prime \prime}\left(r_{H}\right)} \frac{1}{r_{*}}\right)^{-1},
$$

where

$$
S_{0}=\frac{4 \pi}{N^{\prime \prime}\left(r_{H}\right)}\left(\tilde{e}_{I} q_{I}-\tilde{f}\left(r_{H}\right)\right)
$$


and $r_{*}=B\left(r_{H}\right) / B^{\prime}\left(r_{H}\right)$ approximately equals to " $\frac{1}{n-2} \cdot$ radius of the black hole" if the higher derivative corrections in the effective action are small. Thus, after considering that ambiguity in $\tilde{f}\left(r_{H}\right)$ becomes very small and for large $r_{*}$ (sometimes, this corresponds to large charges), the entropy function method gives us an approximate entropy for near-extremal black holes, but the attractor mechanism will be destroyed [15]. In addition, it is also interesting to discuss the extremal rotating black holes with the procedure developed in this paper. Certainly, in this case, the Killing vector which generates the horizon should be of the form $\chi=\partial_{t}+\Omega_{H} \partial_{\phi}$ instead of $\xi=\partial_{t}$. A term including angular-momentum $J$ will naturally appear in the associated entropy function [32]. This issue is under investigation.

\section{Acknowledgements}

L.M.Cao thanks Hua Bai, Hui Li, Da-Wei Pang, Ding Ma, Yi Zhang and Ya-Wen Sun for useful discussions and kind help. This work is supported by grants from NSFC, China (No. 10325525 and No. 90403029), and a grant from the Chinese Academy of Sciences.

[1] S. Ferrara, R. Kallosh and A. Strominger, "N=2 extremal black holes," Phys. Rev. D 52, 5412 (1995) arXiv:hep-th/9508072.

[2] A. Strominger, "Macroscopic Entropy of $N=2$ Extremal Black Holes," Phys. Lett. B 383, 39 (1996) arXiv:hep-th/9602111.

[3] S. Ferrara and R. Kallosh, "Supersymmetry and Attractors," Phys. Rev. D 54, 1514 (1996) arXiv:hep-th/9602136].

[4] S. Ferrara and R. Kallosh, "Universality of Supersymmetric Attractors," Phys. Rev. D 54, 1525 (1996) arXiv:hep-th/9603090.

[5] M. Cvetic and D. Youm, "Dyonic BPS saturated black holes of heterotic string on a six torus," Phys. Rev. D 53, 584 (1996) arXiv:hep-th/9507090.

[6] M. Cvetic and A. A. Tseytlin, "Solitonic strings and BPS saturated dyonic black holes," Phys. Rev. D 53, 5619 (1996) arXiv:hep-th/9512031.

[7] G. Lopes Cardoso, B. de Wit and T. Mohaupt, "Corrections to macroscopic supersymmetric black-hole entropy,” Phys. Lett. B 451, 309 (1999) arXiv:hep-th/9812082.

[8] G. Lopes Cardoso, B. de Wit and T. Mohaupt, "Deviations from the area law for supersymmetric black holes," Fortsch. Phys. 48, 49 (2000) arXiv:hep-th/9904005]. 
[9] G. Lopes Cardoso, B. de Wit and T. Mohaupt, "Macroscopic entropy formulae and nonholomorphic corrections for supersymmetric black holes," Nucl. Phys. B 567, 87 (2000) arXiv:hep-th/9906094.

[10] G. Lopes Cardoso, B. de Wit, J. Kappeli and T. Mohaupt, "Stationary BPS solutions in N = 2 supergravity with $\mathrm{R}^{* *} 2$ interactions," JHEP 0012, 019 (2000) arXiv:hep-th/0009234.

[11] S. Ferrara, G. W. Gibbons and R. Kallosh, "Black holes and critical points in moduli space," Nucl. Phys. B 500, 75 (1997) arXiv:hep-th/9702103.

[12] K. Goldstein, N. Iizuka, R. P. Jena and S. P. Trivedi, "Non-supersymmetric attractors," Phys. Rev. D 72, 124021 (2005) arXiv:hep-th/0507096.

[13] P. K. Tripathy and S. P. Trivedi, "Non-supersymmetric attractors in string theory," JHEP 0603, 022 (2006) arXiv:hep-th/0511117.

[14] K. Goldstein, R. P. Jena, G. Mandal and S. P. Trivedi, "A C-function for non-supersymmetric attractors," JHEP 0602, 053 (2006) [arXiv:hep-th/0512138].

[15] A. Dabholkar, A. Sen and S. P. Trivedi, "Black hole microstates and attractor without supersymmetry," arXiv:hep-th/0611143.

[16] A. Sen, "How does a fundamental string stretch its horizon?," JHEP 0505, 059 (2005) arXiv:hep-th/0411255.

[17] A. Sen, "Stretching the horizon of a higher dimensional small black hole," JHEP 0507, 073 (2005) arXiv:hep-th/0505122.

[18] A. Sen, "Black hole entropy function and the attractor mechanism in higher derivative gravity," JHEP 0509, 038 (2005) arXiv:hep-th/0506177.

[19] A. Sen, "Entropy function for heterotic black holes," JHEP 0603, 008 (2006) arXiv:hep-th/0508042].

[20] B. Sahoo and A. Sen, "BTZ black hole with Chern-Simons and higher derivative terms," JHEP 0607, 008 (2006) arXiv:hep-th/0601228.

[21] B. Sahoo and A. Sen, "Higher derivative corrections to non-supersymmetric extremal black holes in N = 2 supergravity," JHEP 0609, 029 (2006) arXiv:hep-th/0603149.

[22] B. Sahoo and A. Sen, "alpha' corrections to extremal dyonic black holes in heterotic string theory," JHEP 0701, 010 (2007) arXiv:hep-th/0608182].

[23] J. R. David and A. Sen, "CHL dyons and statistical entropy function from D1-D5 system," JHEP 0611, 072 (2006) arXiv:hep-th/0605210.

[24] P. Prester, "Lovelock type gravity and small black holes in heterotic string theory," JHEP 0602, 039 (2006) arXiv:hep-th/0511306. 
[25] M. Alishahiha and H. Ebrahim, "Non-supersymmetric attractors and entropy function," JHEP 0603, 003 (2006) arXiv:hep-th/0601016.

[26] M. Alishahiha and H. Ebrahim, "New attractor, entropy function and black hole partition function," JHEP 0611, 017 (2006) arXiv:hep-th/0605279.

[27] B. Chandrasekhar, S. Parvizi, A. Tavanfar and H. Yavartanoo, "Non-supersymmetric attractors in R**2 gravities," JHEP 0608, 004 (2006) arXiv:hep-th/0602022].

[28] G. Exirifard, "The alpha' stretched horizon in heterotic string," JHEP 0610, 070 (2006) arXiv:hep-th/0604021].

[29] B. Chandrasekhar, "Born-Infeld corrections to the entropy function of heterotic black holes," arXiv:hep-th/0604028.

[30] A. Sinha and N. V. Suryanarayana, "Extremal single-charge small black holes: Entropy function analysis," Class. Quant. Grav. 23, 3305 (2006) arXiv:hep-th/0601183.

[31] A. Sinha and N. V. Suryanarayana, "Two-charge small black hole entropy: String-loops and multi-strings," JHEP 0610, 034 (2006) arXiv:hep-th/0606218.

[32] D. Astefanesei, K. Goldstein, R. P. Jena, A. Sen and S. P. Trivedi, "Rotating attractors," JHEP 0610, 058 (2006) arXiv:hep-th/0606244.

[33] G. L. Cardoso, V. Grass, D. Lust and J. Perz, "Extremal non-BPS black holes and entropy extremization," JHEP 0609, 078 (2006) arXiv:hep-th/0607202].

[34] J. F. Morales and H. Samtleben, "Entropy function and attractors for AdS black holes," JHEP 0610, 074 (2006) arXiv:hep-th/0608044.

[35] D. Astefanesei, K. Goldstein and S. Mahapatra, "Moduli and (un)attractor black hole thermodynamics," arXiv:hep-th/0611140.

[36] B. Chandrasekhar, H. Yavartanoo and S. Yun, "Non-Supersymmetric attractors in BI black holes," arXiv:hep-th/0611240.

[37] G. L. Cardoso, B. de Wit and S. Mahapatra, "Black hole entropy functions and attractor equations," arXiv:hep-th/0612225.

[38] R. G. Cai and D. W. Pang, "Entropy function for 4-charge extremal black holes in type IIA superstring theory," Phys. Rev. D 74, 064031 (2006) arXiv:hep-th/0606098.

[39] R. G. Cai and D. W. Pang, "On entropy function for supersymmetric black rings," arXiv:hep-th/0702040.

[40] R. G. Cai and D. W. Pang, "Entropy function for non-extremal black holes in string theory," arXiv:hep-th/0701158.

[41] M. R. Garousi and A. Ghodsi, "On Attractor Mechanism and Entropy Function for Non- 
extremal Black Holes/Branes," arXiv:hep-th/0703260.

[42] A. Sen, "Extremal Black Holes in String Theory", Lecture in 1st Asian Winter School for String theory, Geometry, Holography and Black Holes, 2007, Korea.

[43] N. V. Suryanarayana and M. C. Wapler, "Charges from Attractors," arXiv:0704.0955] .

[44] R. M. Wald, "Black hole entropy is the Noether charge," Phys. Rev. D 48, R3427 (1993) [arXiv:gr-qc/9307038].

[45] V. Iyer and R. M. Wald, "Some properties of Noether charge and a proposal for dynamical black hole entropy," Phys. Rev. D 50, 846 (1994) arXiv:gr-qc/9403028.

[46] V. Iyer and R. M. Wald, "A Comparison of Noether charge and Euclidean methods for computing the entropy of stationary black holes," Phys. Rev. D 52, 4430 (1995) arXiv:gr-qc/9503052.

[47] S. Gao and R. M. Wald, "The "physical process" version of the first law and the generalized second law for charged and rotating black holes," Phys. Rev. D 64, 084020 (2001) arXiv:gr-qc/0106071].

[48] S. Gao, "The first law of black hole mechanics in Einstein-Maxwell and Einstein-Yang-Mills theories," Phys. Rev. D 68, 044016 (2003) [arXiv:gr-qc/0304094].

[49] C. Teitelboim, "Action and entropy of extreme and nonextreme black holes," Phys. Rev. D 51, 4315 (1995) [Erratum-ibid. D 52, 6201 (1995)] arXiv:hep-th/9410103].

[50] S. W. Hawking, G. T. Horowitz and S. F. Ross, "Entropy, Area, and black hole pairs," Phys. Rev. D 51, 4302 (1995) arXiv:gr-qc/9409013.

[51] G. W. Gibbons and R. E. Kallosh, "Topology, entropy and Witten index of dilaton black holes," Phys. Rev. D 51, 2839 (1995) arXiv:hep-th/9407118. 